(b) If the degree of concentration is higher at the seat of lesion, we shall have a migration of phagocytes thereto.

(c) If the degree of concentration is higher in the blood, the phagocytes may migrate back into the blood.

This teaches us nothing as to the acquisition of immunity, for (1) if true, it is simply a statement of concomitant facts ; (2) on such a view, our successes in the production of immunity become merely a matter of chance and good fortune; (3) it supposes, without the slightest foundation, that in ill animal immunised against a microbe, the toxine of such microbe circulates in its vessels ; and, lastly, it neglects the fact that besides diapedesis, we have, as a rule, hand in hand with positive chemotaxis, a great general increase of phagocytes and leucocytes, depending of necessity on a stimulation not of the leucocytes and phagocytes but of the elements giving birth to these bodies. Hertwig in his monograph sums up this theory in the following manner: " $(a)$ In an immunised organism, the negative chemotaxis, which the body exhibited before being made immune, has been replaced by a chemotaxis behaving positively towards the specific virus." If so, we ask why and how? We are also told that "the effects of the metabolic products of microbes show themselves as positive and negative chemotropism and also as acquired irritability (Reiznachwirkung). The existence of positive chemotropism explains the localisation of the virus through the attraction of leucocytes and subsequent phagocytosis. The existence of negative chemotropism explains the possibility of a general infection by means of a diffusion of the micro-organisms. By means of suitable injections of bacillary products a negative may be changed into a positive chemotaxis, and thus a cure be effected." This is mystical and unsatisfactory, and treats of the body as though it were a specimen on the microscopic slide.

III. We now come to the last point, phagocytosis. I repeat that I fully acknowledge that this exists, and also that it is an important phenomenon during and after the acquisition of immunity, but I am far from accepting that it is the sole, or even the chief, factor active in all cases alike. The exclusive phagocytic theory is both inadequate and unsatisfactory. I will not repeat the classical objections which are always answered by the same classical arguments, but shall enumerate a few difficulties as they have presented themselves to me.

(1) By causıng fever artificially in a susceptible animal two to three hours after the temperature has began to rise considerably there is a very great increase of leucocytes, not only of the so-called lymphocytes but also of the larger elements, and this leucocytosis persists for more than twelve hours. On inoculating the animal when in this condition with anthrax, it will die just as usual. Yet here the army of phagocytes was increased at least tenfold.

(2) Thus the increased number of phagocytes per se is not sufficient. They require also the special training. And here we come to the most unsatisfactory part of the theory. Before an animal is immunised the phagocytes are more or less inert against a particular microbe, but as soon as ever a relative immunity is established they are extremely voracious. We must ask, why does phagocytosis appear now or why are the cells suddenly enabled to destroy what previously perhaps killed them? Metschnikoff says by means of a kind of special selection or change of habit. This to my mind amounts to saying that the cells themselves have acquired immunity: and phagocytosis, instead of explaining the processes by which immunity is acquired, becomes simply a phenomenon observed during or after the process. We must also remember that in many cases after the protective inoculation we have to wait from four to sixteen days before immunity is apparent, and during this time the natural resistance may even be decreased, as Fränkel's experiments on diphtherial immunity show. Leucocytes and wandering cells are but short-lived, and as far as we know constantly replaced through continuous division of cells which form the elements of certain tissues and organs. Thus the assumed hereditary resistance must be sought at the birth-place of these leucocytes and wandering cells. If we allow so much, we might as well say the phagocytes are immune because the whole body has become immune, or simultaneously with the immunisation of the organism, phagocytosis appears.
(3) The occurrence and importance of vaccine fever during the process of immunisation points to more general changes. than a mere change of habit of the phagocyte alone.

(4) That changes exist in all the tissues has been shown by Emmerich, who succeeded to protect animals by inoculating: them with the tissue juices of immunised animals.

(5) Some of the methods of immunisation, as the injection of so-called antitoxine, or of small repeated doses of poison, etc., point strongly to general changes in the body, the phagocytes being altered with the rest of the body.

(6) Lastly, phagocytosis does by no means explain many of the phenomena mentioned in the first part of these remarks. Other objections might be raised, but $I$ am afraid $I$ have been using up time too much already.

In conclusion, it seems to me that we cannot hold any one process exclusively responsible for the artificial production of immunity. The primary cause of immunity, however, must be sought beyond the leucocyte or phagocyte, and also beyond the fluids of the body and, according to the maxims of cellular pathology, depend on changes in the cell-life of the organism which at present we are unable to understand. It is in consequence of these as yet obscure molecular changes that phagocytosis and the other phenomena observed after or during immunisation appear, but none of these alone can be considered the cause of immunity.

\section{NOTE ON}

\section{THE USE OF THE CONSTANT ELECTRIC CURRENT IN THE TREATMENT OF} INTESTINAL OCCLUSION. ${ }^{1}$

BY M. SEMMOLA, M.D.,

Professor of Therapeutics, and Director of the Therapeutical Clinic in theUniversity of Naples; Senator of the Kingdom of Italy.

THE clinical case which forms the subject of this note is very important, and perhaps unique, in medical literature, as demonstrating clearly (1) that there may be an intestinal occlusion due exclusively to transient intestinal paralysis through defective innervation; (2) that the constant electrical current has a truly marvellous effect in these cases.

The patient (C. S., of Secondigliano) was a young man aged 20 , of sound constitution, of normal osseous development, and in good general health, with the exception of a nervous temperament. He fell ill with severe stercoraceous colic, of which he was cured by ordinary treatment. After the colic he suffered from typhlitis and perityphlitis, but was completely cured by antiphlogistic treatment and mills diet. During convalescence he was attacked one day with diarrhœa in consequence of some trivial error in diet. The day after the cessation of the diarrhœa he was attacked with most acute pain, with constipation, persistent vomiting, scantiness of urine, etc. The attacks of pain succeeded each other with great intensity every twenty to thirty minutes, and during these colicky attacks the intestinal coils were clearly visible all over the abdomen. The physicians in attendance instituted very active treatment-hypodermic injections of morphine, ice to the belly, hot hip-baths, poultices, calomel in large doses, etc. All these measures proved futile; the bowels remained locked, the pains continued very severe, the vomiting was obstinate and refractory to treatment, while the scanty secretion of urine was followed on the second day by complete retention, so that the catheter had to be employed two or three times a day. One of the doctors in attendance insisted on using enemata of olive oil, and first two and then three litres were thrown into the bowel. Nevertheless the condition of the patient became worse.

I was called into consultation on the third day, when taking into account, first, the sudden onset of the pain; secondly, the paroxysmal character of the pain, and the freedom from suffering between the attacks when the abdomen was soft and pressure did not cause any pain ; thirdly, the mapping out of the intestinal coils at different points during every attack of pain ; fourthly, the intestinal occlusion which had come on suddenly after the patient had been repeatedly

1 Read in the Section of Medicine at the Annual Meeting of the British Medical Association at Bournemouth. 
purged, and on the day following an attack of acute diarrhœa; fifthly, the existence of paralysis of the bladder, which had come on without any apparent cause so far as the genito-urinary apparatus was concerned, and which has never been recorded as a concomitant of ordinary intestinal occlusion; sixthly, the neurotic temperament of the patient-I distinctly expressed the opinion that the intestinal occlusion was due to nervous paralysis, and I urgently recommended the immediate application of the constant electric current. This advice was at once endorsed by the excellent practitioner in attendance, Dr. D'Auria, but the other medical men maintained that this measure was useless, and that it was necessary to perform laparotomy without delay. Dr. Vizioli, Professor of Electro-Therapeutics in the University of Naples, was called in. The constant current which was employed was furnished by a Daniell's battery with Onimus's piles, modified as regards the graduation and the number of piles by Professor Vizioli. The intensity was measured by a milliampère galvanometer, the strength employed being 10 milliamperes at every application. The positive pole was, by means of a rectal catheter, carried 20 to 25 centimetres up the bowel; and the negative pole, which was olivary in form and covered with cloth steeped in a saturated solution of chloride of sodium, was rubbed transversely over the surface of the abdomen, especially in the parts corresponding to the cæcum, the ascending, transverse, and descending colon, and the sigmoid flexure, as well as over the hypogastric region. The duration of each application was from eight to ten minutes, and there were three sittings every day. By the end of the first day the retention ceased, the patient was able to pass water freely, his general condition improved, especially subjectively, and the attacks of pain were less violent, but the bowels were still constipated.

The surprising result of the treatment shown in the cure of the retention confirmed me in my own opinion of the nature of the case; and notwithstanding pressure constantly brought to bear in the opposite sense by the other doctors, who terrified the family with pictures of imaginary dangers because they were determined at all hazards to have laparotomy performed, the electrical applications were continued, and, after the ninth sitting, the patient had spontaneous motions of the bowels, and by degrees he completely recovered.

\section{A CONTRIBUtION TO THE PHYSICS OF THE ELECTRIC BATH.}

BY W. S. HEDLEY, M.D., Brigade-Surgeon (Retired).

I HAVE lately tried by direct experiment to throw light on some of the physical and physiological problems involved in the administration of the electric bath. Of course, in'entering upon an investigation of this kind, our first solid standpoint must be measurement. In the case before us this almost resolves itself into the question, "What proportion of the main current passes through the human body when immersed under given conditions in an electric (dipolar) bath? This is a primary question but a very complex one. It covers a great deal of ground, and in glancing over the literature of the subject, there appears a tendency to "talk round" this point rather than to approach it by actual experiment.

For the present purpose the following are selected from a large number of experiments bearing on the point. Apparatus used: Oak bath $6 \mathrm{ft}$. long $2 \mathrm{ft} .6 \mathrm{in}$. wide at widest part, the waste pipe being insulated from earth by a short length of rubber pipe inserted near the bath. Battery of $\mathbf{7 4}$ Leclanché cells giving E.M.F. of 75 volts, dipolar bath one electrode $(30 \times 20$ sq. cm. $)$ resting at each end; plain tap water $12 \frac{1}{2}$ in. in bath before immersion of subject; temperature $98^{\circ} \mathrm{F}$.; $\mathbf{R}$. of bath water before entry of subject $165 \mathrm{ohms}^{1}$; subject lying in water, having head resting on strap out of water, and body from shoulders downwards completely immersed, shoulders 8 in. from + electrode, feet 3 in. from - electrode.

Experiment 1.-A current of $100 \mathrm{ma}$. passed through the

$1 R_{0}$ of bath water, taken as it cooled (by ;Wheatstone bridge), at $92^{\circ} \mathrm{F}$ $191 \mathrm{ohms}$, at $87^{\circ}$ F. $2310 \mathrm{~lm} 3$, at $70^{\circ}$ F. 410 ohms. bath. Subject distinctly felt the current as a tingling sensation in his legs but not in other parts of the body. An intercepting current now arranged having one electrode (336 sq. cm.) on nape of neck, the other (95 sq. cm.) on ball of great toe and behind it, this portion of the foot being raised out of the water for the purpose. These electrodes were connected with reflecting galvanometer, having $R$. of 976 ohms. Deflection right off scale but estimated at 500 microampères.

Experiment 2.-Intercepting current arranged with a plain handle electrode of convenient size held in subject's mouth and making good contact with mucous membrane; the other electrode (22.5 sq. cm.) watertight on calf; Edelmann's suspension galvanometer (R. $200 \mathrm{ohms}$ ) ; other conditions as in last experiment. Deflection 0.75 ma. $=(750$ micamp. $)$; constant whilst it lasted for two minutes.

Experiment 3.- Whilst the circuits were still complete the watertight covering of calf electrode was intentionally ruptured, so that the bath water came in contact with the electrode. Deflection 2.5 ma. $=2,500$ micamp.

These experiments seem to afford some direct evidence of the proportion of C. passing through the patient's body in dipolar bath. The readings were carefully taken, twice verified, and found constant after $\mathbf{C}$. had been passing for two minutes. They were further confirmed by an observation with alternating current and telephone. I then proceeded to test the point in other ways. After a simple experiment merely to emphasise (under conditions identical with those of the other experiments) the well-known fact that the chief lines of force do run through the bath water by the shortest and most direct route when not interfered with; the body of the subject was immersed, lying in the direct line of $C$. flow, as in the ordinary way of administering an electric bath, and the results were a striking contrast. Thus, without the body in the water 44 per cent. of total C. was intercepted in the direct line of $\mathrm{C}$. flow, and only 40 per cent. at the sides. The presence of the body in the water caused this condition of things to be reversed, 30 per cent. only being intercepted in the direct line and 48 per cent. at the sides. This seems to point to the fact that the effect of the presence of the patient's body in the water is to deflect to a large extent the lines of force, so that the greater quantity of $\mathrm{C}$. passes round him and not through him.

The next step was to divide the hydro-electric field into sections constituting shunt cireuits (arranged as in following diagram), which could be separately tested without the body and with it.

Results shown in following table:-

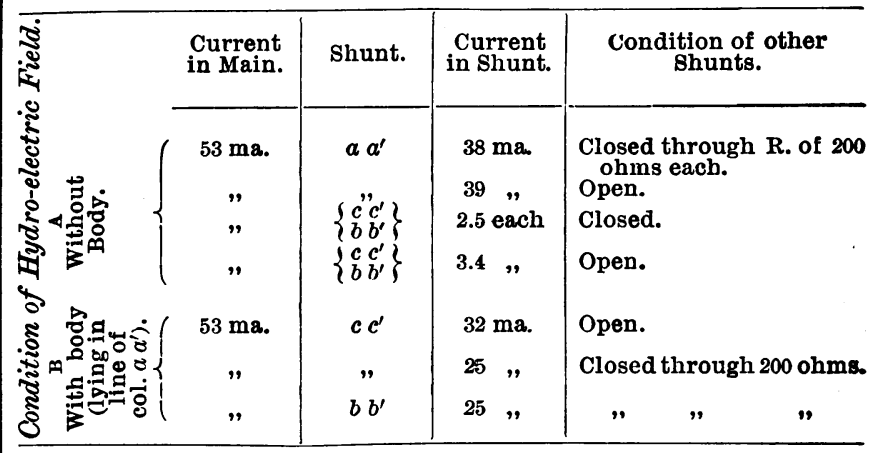

The R. of patient's body, on emerging from the bath, after 5 minutes' immersion-one electrode ( $8 \times 12$ jnches) on shoulder and standing on the other (taken by Wheatstone bridge), was $8,000 \mathrm{ohms}, \mathrm{R}$. of shunt was $200 \mathrm{ohms-the} \mathrm{C.}$ through the shunt $39 \mathrm{ma}$. May we then apply the law of divided circuits as follows :-

$$
\mathrm{X}=\frac{200 \times 39}{8000}=0.97
$$

Therefore 0.97 ma. has passed through the patient's body.

A further consideration of this experiment shows that, as shunt $a a^{\prime}$ gave $38 \mathrm{ma}$., and the two side shunts gave $2.5 \mathrm{each}$, $38+(2.5 \times 2)=43$; and as the total main C. was 53, this leaves 10 as loss by diffusion. When the body was immersed the side shunts gave 25 ma. each ; thus the utmost the body could 